\title{
Evolución del fitoplancton en el Parque Nacional de las Tablas de Daimiel (Ciudad Real, 1996-2002)
}

\author{
Marie Lionard, Elizabeth Ortega Mayagoitia*, Matilde Segura y Carmen Rojo \\ Instituto Cavanilles de Biodiversidad y Biología Evolutiva, Universidad de Valencia. Apartado Oficial \\ 22085. E-46071 Valencia, España. \\ *Facultad de Estudios Superiores de Iztacala. Universidad Nacional Autónoma de México. 54090 \\ Tlalnepantla, México
}

\section{RESUMEN}

El Parque Nacional de Las Tablas de Daimiel (PNTD) es un humedal semiárido situado en la Llanura Manchega (Ciudad Real). En este artículo se expone la dinámica del fitoplancton desde 1996 hasta 2002, examinando las continuidades y divergencias que se observan en el espacio y el tiempo. Tres puntos del humedal (PG: entrada al Parque; MM y PN: salida en zona media y final) se muestrearon mensualmente desde Marzo 1996 a Diciembre 2002. El biovolumen total del fitoplancton del PNTD fue muy variable tanto en el tiempo como en el espacio, el valor mínimo y el máximo se presentaron en PG $\left(0.06 \mathrm{~mm}^{3} / 1\right.$ en mayo de 1996, y $775 \mathrm{~mm}^{3} / 1$ en septiembre de 2001). Se observa un marcado incremento del promedio de biovolumen de todo el humedal a lo largo del tiempo, de $11 \pm 3 \mathrm{~mm}^{3} / 1$ en 1996 a $40 \pm 9 \mathrm{~mm}^{3} / 1$ en 2002. La contribución relativa de los grupos taxonómicos al biovolumen total de fitoplancton mostró también cambios a corto y largo plazo, y fueron distintos en cada una de las tablas muestreadas. Hemos encontrado algunas especies de algas que son indicadoras de cierto grado de salinidad en el agua, ellas son: Chaetoceros muelleri, Urosolenia sp. y Katodinium fungiforme. Así como un aumento en el número de especies y densidad de las euglenofitas. Esta larga serie de datos nos permite extraer una serie de conclusiones sobre el funcionamiento de los productores primarios planctónicos en un humedal somero: a) a la escala espacial de todo el humedal el biovolumen de fitoplancton responde al clima local; b) la tendencia de eutrofización es claramente detectada en todo el humedal; c) las fluctuaciones hidrológicas provocan variaciones a corto plazo, permitiendo una serie continua de estados alternantes y no cíclicos de la comunidad; d) la heterogeneidad espacial de este sistema de islas y tablazos permite una amplia riqueza en el conjunto del humedal.

Palabras clave: humedal, eutrofización, serie temporal larga, heterogeneidad espacio-temporal

\begin{abstract}
Las Tablas de Daimiel National Park (TDNP) is a wetland located in La Mancha Plain (Ciudad Real, Central Spain). Our study reports 1996-2002 phytoplankton dynamics, examining spatio-temporal patterns of continuity and divergence. Three wetland sites (PG-main inlet, MM and PN-main outlets) were sampled monthly from March 1996 to December, 2002. Overall phytoplankton biovolume were very variable in space and time. Extreme values occurred in PG in May, 1996 (0.06 mm3/l) and September, $2001\left(775 \mathrm{~mm}^{3} / \mathrm{l}\right)$. Average values of phytoplankton biovolume experienced aa marked increment over timefrom $11 \pm 3 \mathrm{~mm}^{3} / \mathrm{l}$ in 1996 () to $40 \pm 9 \mathrm{~mm}^{3} / \mathrm{l}$ in 2002. The relative contribution to the total biovolume of the taxonomic groups also showed short and long-term changes and they were different in each of the sampled sites. Some species indicating increasing salinity, such as Chaetoceros muelleri, Urosolenia $s p$. and Katodinium fungiforme, were recorded. Species numbers and density of euglenoids increased as well. This long-term dataset enabled us to draw some conclusions over planktonic primary producers functioning in a shallow wetland: a) at the wetland scale phytoplankton biovolum responds to local climate, b) a eutrophication trend is clearly detected in the whole wetland, c) hydrologic fluctuations are responsible for a short-term variability, thus allowing a variety of non-cyclic assemblage's alternate states, d) spatial heterogeneity arising from the distribution of water tables and islands results in a remarkable species richness at the wetland scale.
\end{abstract}

Keywords: wetland, eutrophication, long-term series, spatial-temporal heterogeneity. 


\section{INTRODUCCIÓN}

Los humedales son ecosistemas acuático-terrestres que por sus características físicas, químicas y biológicas tienen una gran importancia ecológica y social. El Parque Nacional de Las Tablas de Daimiel es un humedal semiárido situado en la Llanura Manchega (Ciudad Real), que alberga una importante diversidad biológica, razón por la cual está inscrito en los convenios Ramsar y ZEPA de conservación (Troya \& Bernùes, 1990). Este humedal sufrió los efectos de una larga sequía que se inició en 1991 y terminó en el verano de 1996, y aunque desde 1997 su régimen hídrico se ha estabilizado, el aumento sostenido de la contaminación de las aguas que lo alimentan ha intensificado el proceso de eutrofización que experimenta (Rubio \& Alvarez-Cobelas, 1996), el cual es reconocible tanto en los períodos de sequía como en los de abundancia de agua (Rojo et al., 1999). Desde hace una década, se lleva a cabo un estudio intensivo de la diversidad y dinámica del fitoplancton en este humedal, abordando de manera paralela la descripción del medio físico y químico, y del resto de componentes del plancton. Los resultados de las investigaciones realizadas hasta 1998 nos han permitido establecer un patrón de la dinámica estacional del plancton, proponiendo mecanismos causa- les (Rojo et al., 2000a), algunos de los cuales han sido comprobados experimentalmente (OrtegaMayagoitia et al., 2002). Continuando con el seguimiento a largo plazo que realizamos en el fitoplancton del humedal, en este artículo se expone la dinámica de estos organismos desde 1996 hasta 2002, examinando las continuidades y divergencias que se observan en el espacio y el tiempo con el objetivo de reafirmar, enriquecer, y/o en su caso, rectificar, el modelo de dinámica estacional del plancton que se ha descrito anteriormente. Esta serie de datos permitirá además describir la trayectoria del componente algal del plancton a lo largo del proceso de eutrofización del humedal.

\section{MATERIALES Y MÉTODOS}

El Parque Nacional de Las Tablas de Daimiel (PNTD) es un humedal hipertrófico constituido por una serie de islas, zonas de macrófitos emergentes y zonas de aguas libres, someras e interconectadas, las cuales tienen dinámicas ecológicas claramente diferenciadas. Hasta hace algunos años, el Parque se alimentaba de las aguas de un acuífero local y de los ríos Gigüela y Guadiana. Sin embargo, hoy en día el río Guadiana ha dejado de manar, siendo el río Gigüela, que transporta

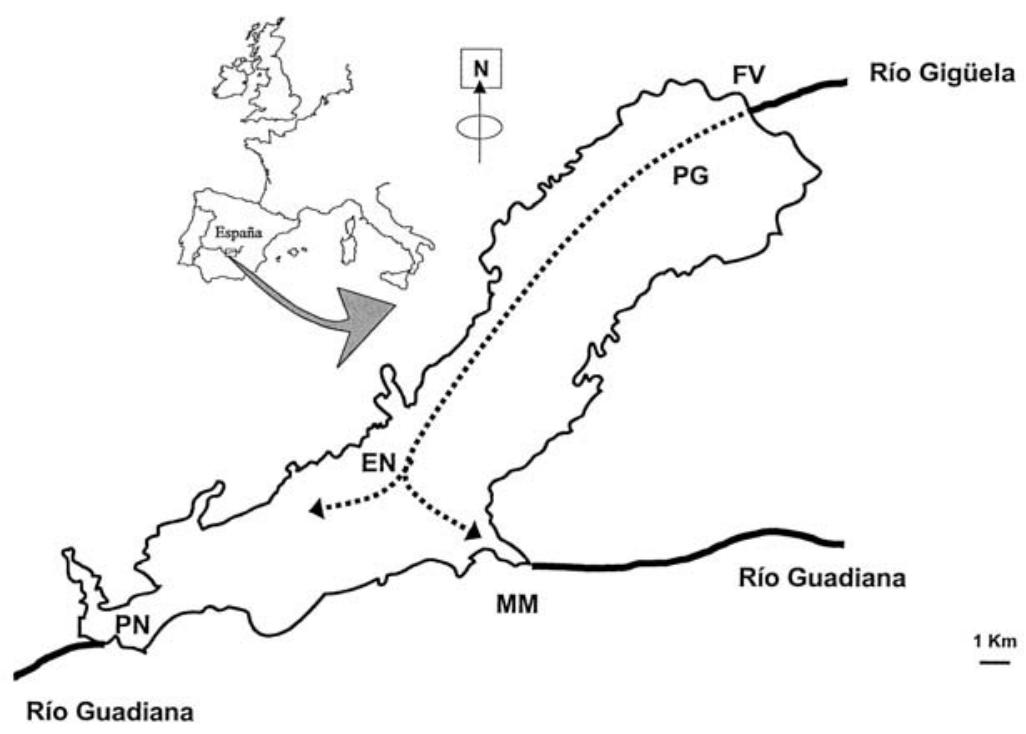

Figura 1. Mapa de las Tablas de Daimiel; se indican los tres puntos de muestreo: Patagallina, Molemocho y Puente Navarro. Map of Tablas de Daimiel; the three sampling points are shown: Patagallina, Molemocho and Puente Navarro. 
aguas provenientes de un trasvase del Tajo-Segura, la principal fuente hídrica del humedal (Fig. 1).

Las muestras de fitoplancton fueron recogidas, mensualmente desde marzo de 1996 hasta diciembre de 2002 en un punto del humedal llamado Molemocho (MM) y en otros dos puntos, Patagallina (PG) y Puente Navarro (PN), el mismo periodo salvo el año 1999. PG es el punto de entrada del canal del río Gigüela en el Parque, esta zona de $1.5 \mathrm{~m}$ de profundidad es fácilmente desbordada cuando entra agua al Parque, por eso a comienzos de 1997 y de 1998 no pudo ser muestreado; MM (2 $\mathrm{m}$ de profundidad) es el punto en el que entraban las aguas del río Guadiana pero actualmente es una zona por la cual drenan las aguas del humedal; PN es el punto de muestreo más profundo $(4 \mathrm{~m})$, en él se construyó una pequeña presa para regular el nivel de inundación del humedal. Todas las muestras se tomaron a unos $25 \mathrm{~cm}$ de la superficie.

Las muestras se fijaron con lugol, haciéndose las observaciones y recuentos mediante el método de Utermöhl, con un microscopio invertido Olympus CK2 a 400 y 1000x (Aboal et al., 1996). La metodología utilizada en la identificación de las microalgas ha quedado reflejada con detalle en los trabajos de Rojo et al. (1999) y Ortega-Mayagoitia \& C. Rojo (2000a y 2000b). El criterio para detener el recuento en cada muestra fue alcanzar por lo menos 100 individuos de la especie más abundante, con lo cual el error estimado para la media es de $\pm 20 \%$. Posteriormente calculamos el biovolumen promedio de cada especie utilizando las fórmulas de figuras estereométricas sugeridas por Rott (1981). Los detalles sobre los cálculos de la densidad y biovolumen del fitoplancton pueden consultarse en Rojo et al. (2000a).

\section{RESULTADOS}

\section{Dinámica temporal del biovolumen de fitoplancton}

El biovolumen total del fitoplancton del PNTD fue muy variable temporal y espacialmente a lo largo de los siete años estudiados. El valor mínimo y el máximo se presentaron en PG: 0.06 $\mathrm{mm}^{3} / 1$ en mayo de 1996 , y $775 \mathrm{~mm}^{3} / 1$ en septiembre de 2001. De manera general, la dinámica anual del fitoplancton puede considerarse estacional, con sus valores de biovolumen más bajos en la primera mitad del año, en ocasiones con un pequeño pico primaveral, y los mayores al final del verano o comienzos del otoño (Fig. 2). Este patrón fue especialmente claro de 1996 a 1998 ,

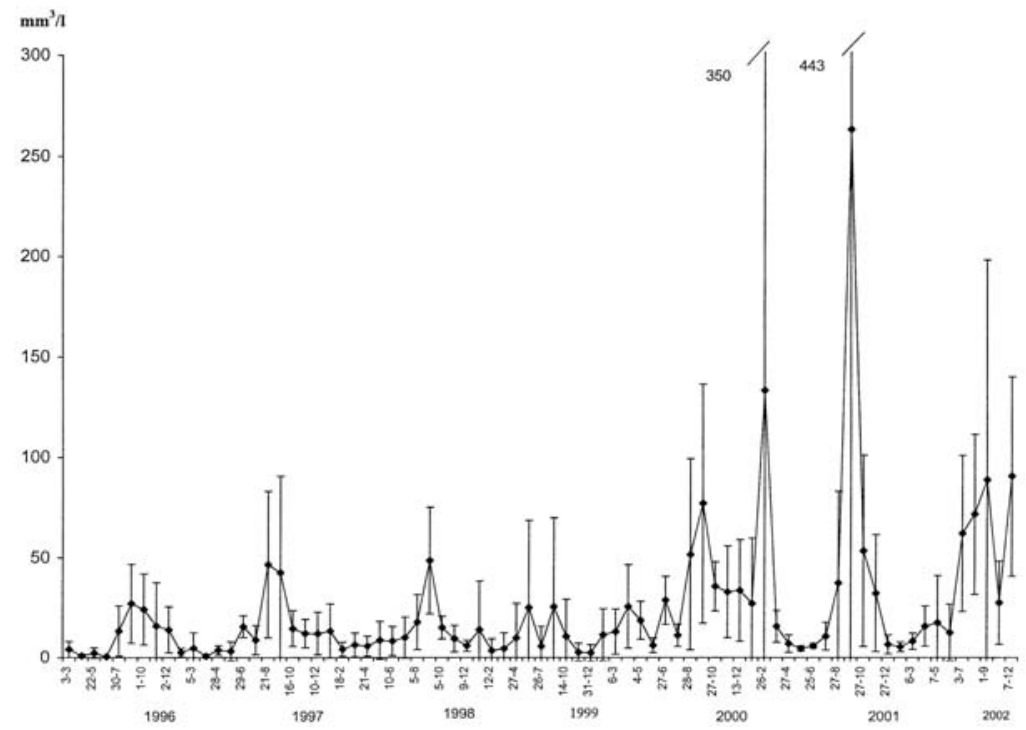

Figura 2. Variaciones del bivolumen medio anual del fitoplancton. La barra indica el error estándar. Variations of mean annual phytoplankton biovolume. Bar represents Standard error. 
pero a partir de 1999 se observaron algunas variaciones importantes (Fig. 3): en la primavera de ese año, en MM - sitio del que se tiene la serie de datos completa - se presentó un pico de biovolumen de la misma magnitud que el de verano; en PN se registró el máximo algal durante el invierno de 2000. Solamente en PG se conservó la pauta descrita para 1996-1998, durante 2000 a 2002, en
MM y PN un aumento del biovolumen en otoño e invierno se empieza a hacer patente hasta el 2002. Por otra parte, el biovolumen total del fitoplancton fluctuó de manera distinta en cada tabla a lo largo del estudio (Fig. 3): en PG se observa un claro patrón estacional con máximos a finales de verano, hecho que se acentúa en los últimos años (del 2000 al 2002) debido a florecimientos masivos de
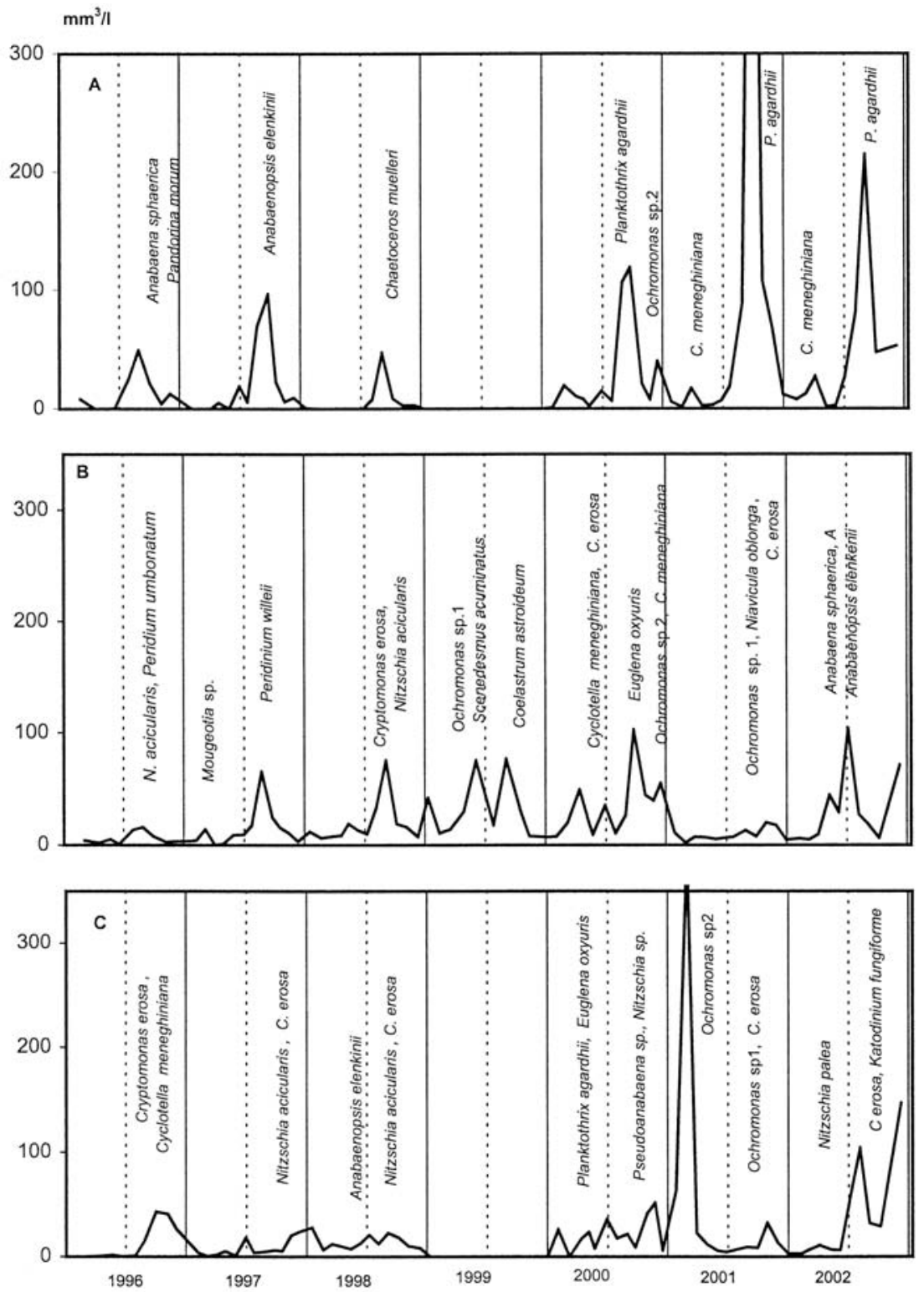

Figura 3. Dinámica del biovolumen de fitoplancton en PG (A), MM (B) y PN (C). Se indican las especies que constituyen como mínimo el $80 \%$ del biovolumen total (solas o como co-dominantes). Phytoplankton biovolume dynamics in PG (A), MM (B) y PN (C). Species which represent a minimum of $80 \%$ of total biovolume are indicated (alone or codominant). 
Planktothrix agardhii. MM presentó un incremente paulatino del biovolumen algal que se interrumpió en 2001, cuando disminuyó de manera importante quedando con valores tan bajos como tenía en 1996 y recuperando sus valores habituales en 2002, su estacionalidad no resulta tan evidente como en PG especies de nostocales dominaron en verano (Anabaena y Anabaenopsis). En PN el biovolumen osciló durante los tres primeros años y aumentó posteriormente, de manera especial durante 2001 debido a Ochromonas en invierno. Estos espectaculares crecimientos de fitoplancton provocaron la observada tendencia de incremento del promedio de biovolumen de todo el humedal (Fig. 2): $11 \pm 3 \mathrm{~mm}^{3} / 1$ en 1996, $14 \pm 4 \mathrm{~mm}^{3} / 1$ en $1997,13 \pm 2 \mathrm{~mm}^{3} / 1$ en $1998,10 \pm 4 \mathrm{~mm}^{3} / 1$ en $1999,29 \pm 5 \mathrm{~mm}^{3} / 1$ en $2000,50 \pm 24 \mathrm{~mm}^{3} / 1$ en 2001, y $40 \pm 9 \mathrm{~mm}^{3} / 1$ en 2002. Aunque globalmente la tendencia es a aumentar, en el año 2002 no se superaron los valores del 2001.

\section{Variación temporal de las poblaciones dominantes}

Las especies algales responsables de los picos de abundancia no fueron las mismas simultáneamente, ni se repitieron cada año (Fig. 3):

En PG las especies responsables de la mayoría de los picos de abundancia veraniegos fueron cianoficeas: Anabaena sphaerica en 1996, Anabaenopsis elenkinii en 1997 y Planktothrix agardhii en 2000, 2001 y 2002. Por su parte, la diatomea Cyclotella meneghiniana presente en los veranos de 1997 y 1998 formó los picos primaverales de 2000, 2001 y 2002.

En MM el conjunto de especies dominantes fue más diverso: en 1996 el máximo biovolumen se observó durante julio y agosto sin llegar a ser un pico propiamente dicho, siendo Nitzschia acicularis y Peridinium umbonatum las especies más abundantes, que aportaron el $47 \%$ y el $38 \%$ al total, respectivamente. Nitzschia acicularis fue co-dominante también en los veranos de 1998 y 1999, para posteriormente casi desaparecer del humedal y ser sustituida en MM por Nitzschia palea (aunque no dominante) en el verano de 2000, y por Navicula oblonga en 2001. Esta dominancia de la diatomeas pennadas en verano ha sido sustituida por una clara dominancia de cianobacterias filamentosas (Anabaenopsis elenkinii y Anabaena sphaerica). Otras especies importantes en los crecimientos de verano fueron Peridinium willei en 1997, Cryptomonas erosa en 1998, y Coelastrum astroideum en 1999, mientras que en primavera sobresalieron Scenedesmus acuminatus en 1999 y Cyclotella meneghiniana en 2000 que también se observa como dominante en los inviernos de 2000 a 2002.

En PN los picos de fitoplancton se desarrollaron principalmente durante los otoños, en ellos Cryptomonas erosa dominó o co-dominó de 1996 a 1998, compartiendo importancia con Cyclotella meneghiniana en el primer año, y con Nitzschia acicularis y Anabaenopsis elenkinii los dos años siguientes. El panorama cambió posteriormente, ya que en 2000 predominaron en verano y otoño las oscillatoriales, mientras que en 2001 se presentó un gran crecimiento en invierno debido a Ochromonas sp. 2, y el posterior crecimiento de otoño lo integró en su mayor parte Ochromonas sp. 1. La creciente importancia de estas crisofíceas se hizo patente también en el resto de las Tablas, en las cuales formó pequeños picos durante la transición del otoño al invierno. En 2002 de nuevo se observa la dominancia de diatomeas pennadas y de Cryptomonas erosa, es en esta Tabla donde la dinámica de las especies dominantes resulta más impredecible (Fig. 3).

\section{Variación temporal de los grupos taxonómicos}

La contribución relativa de los grupos taxonómicos al biovolumen total de fitoplancton mostró también cambios a corto y largo plazo, y fueron distintos en cada una de las tablas muestreadas. En PG no se observó un patrón de composición claro durante los primeros tres años: en 1996 los grupos más importantes fueron sucesivamente diatomeas (invierno), clorofíceas (primavera), cianofíceas (verano) y criptofíceas (otoño), disminuyendo en los siguientes dos años la contribución de las cianofíceas, y 

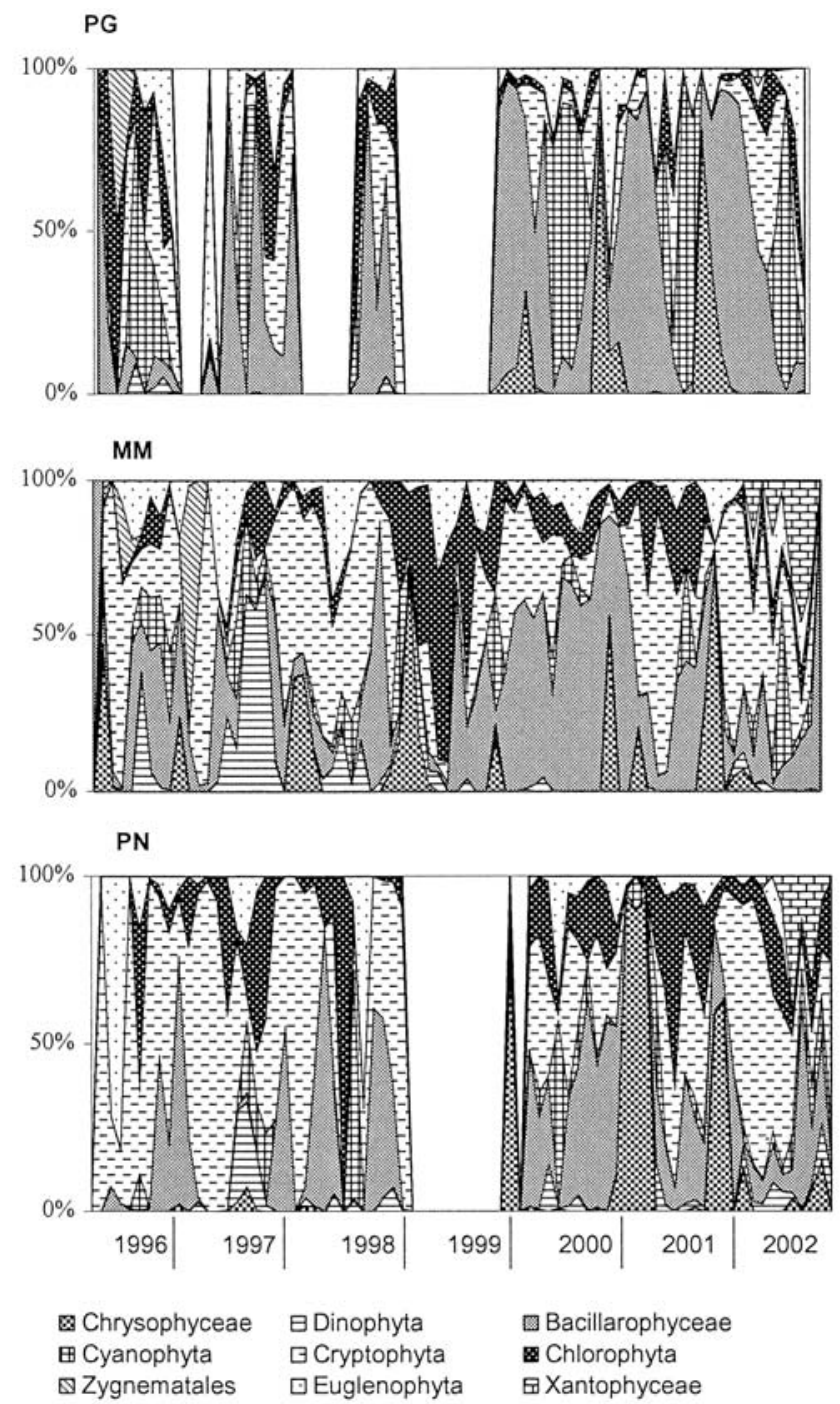

Figura 4. Dinámica de la contribución relativa de los grupos taxonómicos al biovolumen total. Se consideran las Chlorophyta sin Zignematales. Dynamics of the relative contribution of taxonomic groups to total biovolume. Chlorophyta do not include Zignematales.

aumentando la de las diatomeas (Fig. 4). Desde 2000 a 2002 se observa un patrón similar: clorofíceas y criptofíceas tuvieron un aporte inferior, el fitoplancton se compuso principalmente de diatomeas durante la primera parte del año, y de cianoficeas durante la segunda, con importantes contribuciones de crisofíceas -antes prácticamente ausentes- al final del otoño.

En MM, se observó un patrón más complejo y variable: las criptoficeas fueron por lo general
(4 de los 7 años estudiados) el grupo taxonómico dominante durante la primera parte del año, y las diatomeas durante la segunda, con picos regulares de crisoficeas al final del otoño $\mathrm{y} / \mathrm{o}$ principios del invierno. Sin embargo, cada año se presentaron variaciones importantes: en 1996, después del pico primaveral de criptofíceas con zignematales (Mougeotia sp. y Spirogyra parva), el fitoplancton de verano estuvo dominado por diatomeas, más una mezcla de 
cianofíceas, dinoflagelados, euglenofíceas, criptofíceas y clorofíceas; el verano siguiente los dinoflagelados fueron el grupo dominante, pero luego disminuyeron su aportación cada año hasta casi desaparecer. En 1998 las criptoficeas aumentaron su contribución durante el invierno y la primavera, extendiéndose también durante el verano y el otoño, para disminuir posteriormente ante incrementos sustanciales de las clorofitas durante 1999, y de las diatomeas en 2000 y hasta inicios del 2001. En ese año se restableció la dominancia de criptofíceas durante la primera mitad del año, para terminar con una mezcla del resto de grupos taxonómicos en verano-otoño. Un patrón similar se observa en 2002 con la novedad de la destacada presencia de xantofíceas (Centritractus sp.).

En PN, las criptoficeas fueron claramente las algas dominantes durante los tres primeros años, aunque cada año los picos mayores del $80 \%$ fueron haciéndose cada vez más aislados y espaciados. Durante el 2000 y 2001 las criptofíceas ya no sobrepasaron el $50 \%$ del biovolumen del fitoplancton en ninguna ocasión, aunque continuaron siendo un componente importante junto con las diatomeas, cianofíceas y clorofíceas. De hecho, durante 2000 y 2001, el único grupo que mostró una clara tendencia estacional fue el de las crisoficeas, que anteriormente habían sido muy escasas, pero que crecieron de manera importante al comienzo del invierno de ambos años, y también en el otoño de 2001. En 2002, el patrón de distribución se parece bastante al de MM, vuelve a tener una mayor presencia de criptofíceas y aparecieró la xantofícea Centritractus sp.

\section{Las asociaciones de especies}

La dinámica de sustitución de unas asociaciones algales por otras se ha establecido en base a las especies dominantes en biovolumen y a la clasificación desarrollada por Reynolds (1997).

La serie de muestras de PG es incompleta de 1996 a 1998, pero en los datos que poseemos, observamos que 1) la asociación H (con componentes como Anabaena, Anabaenopsis) se pre- sentó de manera regular en verano aunque cada año con compañeros diferentes (G -Eudorinaen 1996, C -Asterionella- en 1997, J -Scenedesmus - en 1998), 2) que en otoño solía ser común la presencia de Y -Cryptomonas- (1996 y 1997) y la de C (de 1997 en adelante), y que asociaciones como la J, G, D (Nitzschia) y $\mathrm{X}_{2}$ (Chrysochromulina) se presentaron, pero de manera desordenada e irregular.

En 2000 y 2001 observamos un patrón sorprendentemente parecido, con la asociación $\mathrm{C}$ al principio del año, sumándosele $\mathrm{D}$ durante la primavera. En el verano entró $\mathrm{H}$ seguida inmediatamente por la muy clara asociación Planktothrix agardhii - Limnothrix sp. (S) al final del verano. Esta asociación es seguida en otoño de un pico de Ochromonas sp. (asociación E) y/o nuevamente la asociación de diatomeas C. En ambos años notamos la presencia intermitente de W (Euglena) e Y. El patrón dominante en estos dos últimos años podría resumirse como $\mathrm{C} \rightarrow \mathrm{C} / \mathrm{D} \rightarrow \mathrm{H} \rightarrow \mathrm{S} \rightarrow \mathrm{E} / \mathrm{C}$.

En MM notamos, entre la primavera y el verano de 1996 a 1998, la sucesión de las asociaciones $\mathrm{Y}, \mathrm{D}, \mathrm{W}$ y L (Peridinium), que son típicas de las aguas ricas en materia orgánica. Sin embargo, el orden de aparición no fue siempre el mismo, y las asociaciones D e Y se observaron también en otoño. En 1999, el invierno estuvo dominado por la asociación E, las criptofitas casi desaparecieron, y las clorofitas de la asociación J incrementaron su importancia de tal manera que la sucesión a lo largo del año fue $\mathrm{E} \rightarrow \mathrm{J} / \mathrm{W} \rightarrow \mathrm{J} / \mathrm{D} \rightarrow \mathrm{D} / \mathrm{W} / \mathrm{Y}$. En el año 2000 el patrón de especies dominantes cambió, de modo que la asociación $\mathrm{C}$, antes casi ausente, dominó desde el invierno hasta el otoño, acompañada de $\mathrm{Y}$ en invierno y primave$\mathrm{ra}$, y por $\mathrm{D}$ en verano y otoño, de la siguiente manera: $\mathrm{C} / \mathrm{Y} / \mathrm{D} \rightarrow \mathrm{C} / \mathrm{Y} \rightarrow \mathrm{C} / \mathrm{D} / \mathrm{W} \rightarrow \mathrm{C} / \mathrm{D} / \mathrm{E}$. Por último, en 2001, C apareció solamente en invierno, y Y recuperó terreno, de modo que la sucesión fue $\mathrm{C} \rightarrow \mathrm{Y} \rightarrow \mathrm{Y} / \mathrm{X} \rightarrow \mathrm{Y} / \mathrm{D} / \mathrm{J} \rightarrow \mathrm{E}$.

En PN observamos que, a diferencia de los otros puntos muestreados, la asociación $\mathrm{Y}$ se presentó de manera continua durante tramos importantes del año, en todos los años de los 

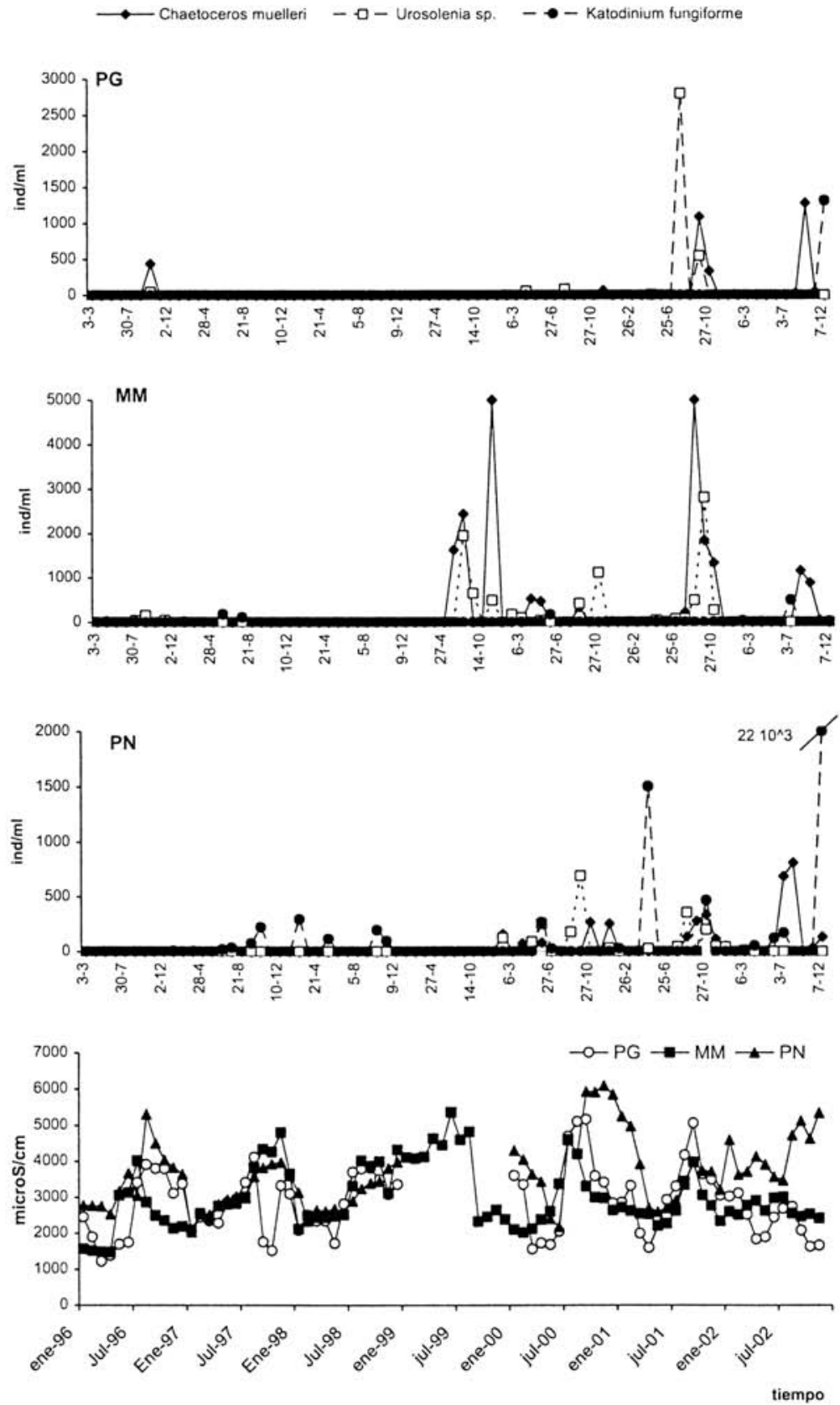

Figura 5. Dinámica de la densidad de tres especies indicadoras de salinidad en los tres puntos de muestreo (A: PG, B: MM y C: PN). Dinámica de la conductividad en los tres puntos (D). Dynamics of density of three salinity indicator species in the three sampling points (A: PG, B: MMy C: PN). Dynamics of conductivity in the three points (D).

que tenemos datos. Sin embargo, otras asociaciones estuvieron presentes, pero de manera más cambiante, además, se presentaron numerosos picos de crecimiento de algas, aunque de manera aislada. En 1996, la sucesión a partir de la primavera fue $\mathrm{Y} / \mathrm{W} \rightarrow \mathrm{Y} \rightarrow \mathrm{Y} / \mathrm{C}$. En el siguiente año, el fitoplancton de invierno estuvo dominado por las diatomeas de las asociaciones $\mathrm{C}$ y D para luego dar paso a algas flageladas: $\mathrm{C} / \mathrm{D} \rightarrow$ $\mathrm{Y} / \mathrm{X}_{2} \rightarrow \mathrm{Y} \rightarrow \mathrm{Y} / 1 / \mathrm{W} / \mathrm{X}_{2} \rightarrow \mathrm{Y}$. En 1998 encontra- 
mos un patrón más sencillo, con $\mathrm{Y}$ dominando completamente en invierno, junto con $\mathrm{C}$ en primavera, y con $\mathrm{D}$ al final del verano y en otoño: $\mathrm{Y} \rightarrow \mathrm{Y} / \mathrm{C} \rightarrow \mathrm{Y} \rightarrow \mathrm{Y} / \mathrm{D}$. El patrón fue similar en 2000 , sólo que entonces la asociación E apareció en el invierno, y las cianofíceas filamentosas (S) aumentaron su importancia, de modo que observamos $\mathrm{E} \rightarrow \mathrm{Y} / \mathrm{C} \rightarrow \mathrm{W} / \mathrm{S} \rightarrow \mathrm{Y} / \mathrm{C} \rightarrow \mathrm{D} / \mathrm{S}$, junto con una mezcla de $\mathrm{Y} / \mathrm{C} / \mathrm{W}$ al final del invierno. En los últimos años de observaciones notamos que la presencia de la asociación $\mathrm{E}$ aumentó considerablemente durante el invierno y el otoño, de manera que la sucesión podría resumirse como $\mathrm{E} \rightarrow \mathrm{Y} \rightarrow \mathrm{Y} / \mathrm{D} \rightarrow \mathrm{E} / \mathrm{Y}$.

\section{Evolución de la presencia de especies indicadoras}

En el humedal de Las Tablas de Daimiel hemos encontrado algunas especies de algas que son indicadoras de cierto grado de salinidad en el agua, ellas son: Chaetoceros muelleri, Urosolenia sp. y Katodinium fungiforme (Lange \& Tiffany 2002, Round et al., 1990). Si bien durante los tres primeros años del estudio su presencia fue escasa en PG y MM, su densidad se incrementó visiblemente a partir de 1999 (Fig. 5). En cada tabla la especie más abundante fue diferente: en PG Urosolenia sp. alcanzó $2800 \mathrm{ind} / \mathrm{ml}$ en el verano de 2001; en MM se observaron varios picos de Chaetoceros muelleri, el más alto de ellos fue de $4800 \mathrm{ind} / \mathrm{ml}$ también en el verano de 2001; por último, en PN la densidad puntual más alta fue de Katodinium fungiforme, con $23000 \mathrm{ind} / \mathrm{ml}$ en diciembre de 2002.

La conductividad en Las Tablas de Daimiel oscila estacionalmente, con valores bajos a principios del año, los cuales se incrementan paulatinamente a lo largo de los meses para luego descender, al final del verano o en otoño (Fig. 5). Se observaron variaciones en los máximos de conductividad de cada año, con $5310 \mu \mathrm{S} / \mathrm{cm}$ en 1996 (PN), $4800 \mu \mathrm{S} / \mathrm{cm}$ en 1997 (MM), $4320 \mu \mathrm{S} / \mathrm{cm}$ en 1998 (MM), $5360 \mu \mathrm{S} / \mathrm{cm}$ en 1999 (MM), alcanzando el valor más alto hasta ahora registrado en 2000 , que es de $6090 \mu \mathrm{S} / \mathrm{cm}(\mathrm{PN})$, para luego descender ligeramente a $5060 \mu \mathrm{S} / \mathrm{cm}$ en 2001

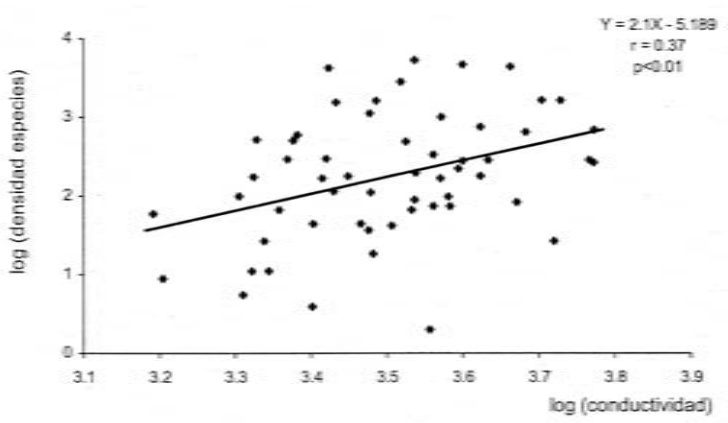

Figura 6. Relación entre conductividad y la densidad total de las poblaciones indicadoras de salinidad $(\mathrm{n}=145)$. Relationship between conductivity and total density of salinity indicator populations $(n=145)$.

(PG), $5350 \mu \mathrm{S} / \mathrm{cm}$ en 2002 (PN). Además, encontramos una correlación directa significativa entre la densidad total de las tres especies indicadoras, y la conductividad (Fig. 6).

Por otra parte, como indicadoras de un aumento en la contaminación por materia orgánica se observa la dinámica de las euglenofitas (Conforti et al. en prensa en este volumen). En 1996, el máximo de las euglenofitas se encuentraba en PG (entrada de agua al humedal) con $310^{3} \mathrm{ind} / \mathrm{ml}$ debido esencialmente a $E$. gracilis $\left(2.810^{3} \mathrm{ind} / \mathrm{ml}\right)$ y había poca densidad en MM y PN (punto intermedio y final del curso del agua por el humedal). Sin embargo, en estos últimos años se encontraron valores muy altos en MM (2 $10^{3} \mathrm{ind} / \mathrm{ml}$ en verano desde 2000) y en PN algún pico (1.5 $10^{3} \mathrm{ind} / \mathrm{ml}$ en enero de 2001).

\section{DISCUSIÓN}

Las Tablas de Daimiel es un humedal en el que se tiene constancia, al menos, de un largo periodo de sequía que acabó en el invierno entre 1996 y 1997, comenzando entonces lo que podríamos llamar su periodo húmedo (SánchezCarrillo et al., 2000). Sin embargo, la mayoría de sus entradas de agua son gracias a los trasvases desde el Tajo que afortunadamente cada vez parecen mejor gobernados. Por todo ello, la dinámica de las comunidades acuáticas está 
sujeta a fluctuaciones hídricas que distan mucho de ser cíclicas (Álvarez-Cobelas et al., 2001).

El estudio a largo plazo de los productores primarios planctónicos nos revela en primer lugar la importancia de los factores abióticos en el control de su dinámica, siendo el clima el determinante de la estacionalidad casi cíclica del biovolumen del fitoplancton como se observa en este trabajo. Ahora bien, la alta heterogeneidad espacio-temporal encontrada se debe explicar por otro factor abiótico que funciona a otra escala: la hidrología (régimen de inundación, superficie inundada, permanencia del agua, etc.). El régimen hídrico resultó ser un buen motor en la dinámica de las microalgas en los primeros años tras la sequía (Angeler et al., 2001, Rojo et al., 2000a) y nos explicaría las fluctuaciones que se encuentran hasta hoy. En tercer lugar observamos un incremento del biovolumen de fitoplancton en el tiempo debido al proceso de eutrofización tanto por el estado trófico del agua que recibe (Cirujano \& Álvarez-Cobelas 1997) como por el fenómeno de carga interna de nutrientes proveniente del sedimento (Sánchez-Carrillo et al., 2000, Sánchez-Carrillo \& Álvarez-Cobelas 2001) y queda bien ilustrado por la relevancia que están adquiriendo las poblaciones indicadoras de estados avanzados de eutrofización (Álvarez-Cobelas \& Jacobsen 1992, Conforti et al. en prensa, en este volumen).

Un proceso que no se había detectado hasta el momento es la salinización. Aunque alguna zona del humedal ha presentado siempre mayor conductividad (MM) y se han observado esporádicamente algunas especies propias de aguas salobres (Ortega-Mayagoitia \& Rojo 2000b). El aumento de estas poblaciones en el Parque en los últimos dos años parece un indicador de una tendencia que aún no se ve clara en la serie temporal de la conductividad y deberá ser analizada en el futuro.

Por otra parte, la situación de sistema somero altamente perturbado se refleja en la rica secuencia de estados alternantes (no alternativos sensu Scheffer 1991) es decir, se pueden observar sustituciones de la estructura de la comunidad que nada tienen que ver con la estacionalidad ni son periódicas, alternancia que ya se descrito en otros lugares someros (Rojo et al., 2000b, Rojo \& Álvarez-Cobelas 2001) y que confirma la idea de Allen et al. (1977) de más estados que estaciones y la de Reynolds (1997) de no retorno a un mismo estado cíclicamente. Si la fluctuación irregular abiótica puede explicarnos la variabilidad de las estructuras de los productores primarios en el tiempo, el hecho de ser un paisaje en mosaico con islas que a veces se conectan nos explicará la alta heterogeneidad espacial que se observa en el plancton (Rodrigo et al., 2002, Shiel et al., 1998). Así, tal y como se ha sugerido en otros paisajes acuáticos someros y se ha demostrado experimentalmente en el caso de Las Tablas de Daimiel (Angeler et al., 2002, Ortega-Mayagoitia et al., 2002) la heterogeneidad morfológica de los cuerpos de agua determina que cada zona de este humedal presente una dinámica del plancton completamente diferente a las otras. Sin embargo, esta misma heterogeneidad permite que la flora sea altamente conservativa, actuando cada tablazo del humedal con su dinámica independiente como un continuo reservorio para las otras zonas, así se observa que desde 1996 las poblaciones relevantes son prácticamente las mismas.

La perspectiva de un estudio a largo plazo nos permite sugerir una serie de conclusiones sobre el funcionamiento de los productores primarios planctónicos en un humedal somero: a) el biovolumen responde a una mayor escala y a un factor abiótico como el clima: patrón estacional común en todo el humedal; b) la eutrofización como tendencia es global en el humedal; c) las fluctuaciones hidrológicas provocan una variabilidad a una escala menor que la estacional permitiendo una serie continua de estados alternantes no cíclicos de la comunidad; d) la heterogeneidad espacial conseguida en el sistema de islas y tablazos permite una amplia riqueza en el conjunto del humedal.

\section{AGRADECIMIENTOS}

Agradecemos a Palmira Riolobos el necesario trabajo de campo. Este estudio se hizo gracias a 
la financiación del Organismo Autónomo de Parques Nacionales (Ministerio de Medio Ambiente) y a la Beca francesa que permitió a Marie Lionard colaborar en este proyecto.

\section{BIBLIOGRAFÍA}

ABOAL M., M. ALVAREZ-COBELAS, M. ARAUZO, M. CARRASCO, C. CASADO, S. CIRUJANO, M. DORADO, B. ELVIRA, J. GARCIA-AVILES, M. GARCIA SANCHEZ-COLOMER, J.L. DE LA HORRA, C. ROJO, A. RUBIO, M.J. SANCHEZ SOLER \& J.L. VELASCO. 1996. Metodologías. En: Las Tablas de Daimiel. Ecología acuática y sociedad. Alvarez-Cobelas M. \& Cirujano S. (eds): 299-305. Ministerio Español de Medio Ambiente, Madrid.

ALLEN, T. F. H., S. M. BARTELL \& J. F. KOONCE. 1977. Multiple stable configurations in ordination of phytoplankton community changes rates. Ecology, 58: 1076-1082.

ALVAREZ-COBELAS M. \& B. A. JACOBSEN. 1992. Hypertrophic phytoplankton: an overview. Freshwater Forum, 2:184-199.

ALVAREZ-COBELAS, M., S. CIRUJANO \& S. SÁNCHEZ-CARRILLO. 2001. Hydrological and botanical man-made changes in the Spanish wetland of Las Tablas de Daimiel. Biological conservation, 97:89-98.

ANGELER, D., M. ÁLVAREZ-COBELAS, C. ROJO \& S. SÁNCHEZ-CARRILLO. 2001. The significance of water inputs to plankton biovolum and trophic relationships in a semi-arid freshwater wetland (central Spain). Journal of Plankton Research, 22: 2075-2093.

ANGELER, D. G., M. ÁlVAREZ-COBELAS, S. SÁNCHEZ-CARRILLO \& M. A. RODRIGO. 2002. Assessment of exotic fish impacts on water quality and zooplankton in a degraded semi-arid floodplain wetland. Aquatic Science, 64: 76-86.

CIRUJANO, S. \& M. ÁLVAREZ-COBELAS. 1997. La contaminación del agua impide la recuperación de Las tablas de Daimiel. Quercus, 139: 42-44.

CONFORTI, V., M. LIONARD \& C. ROJO. Las euglenofitas en Las Tablas de Daimiel: indicadoras de la degradación de un humedal. Limnetica, (en prensa).

LANGE, C. B. \& M. A. TIFFANY. 2002. The diatom flora of the salton sea, California. Hydrobiologia, 473: 179-201.
ORTEGA-MAYAGOITIA, E. \& C. ROJO. 2000a. Fitoplancton del Parque Nacional Las Tablas de Daimiel. II Las cianofíceas, los dinoflagelados, las criptofíceas, las crisofíceas y las xantofíceas. Anales del Jardín Botánico de Madrid, 57:251-266.

ORTEGA-MAYAGOITIA, E. \& C. ROJO. 2000b. Fitoplancton del Parque Nacional Las Tablas de Daimiel. III Diatomeas y clorofitas. Anales del Jardín Botánico de Madrid, 58:17-37.

ORTEGA-MAYAGOITIA, E., C. ROJO \& M. A. RODRIGO. 2002. Factors masking the trophic cascade in shallow eutrophic wetlands: evidence from a microcosm study. Arch. Hydrobiol., 155: 43-63.

REYNOLDS, C. S. 1997. Vegetation processes in the pelagic: a model for ecosystem theory. Ecology Institute, Oldendorf-Luhe.

RODRIGO, M. A., C. ROJO \& X. ARMENGOL. 2002. Plankton biodiversity in a landscape of shallow water bodies (Mediterranean coast, Spain). Hydrobiologia, (en prensa).

ROJO C., E. ORTEGA-MAYAGOITIA \& V. CONFORTI. 1999. Fitoplancton del Parque Nacional de las Tablas de Daimiel. I Las Euglenofitas. Anales del Jardín Botánico de Madrid, 57:15-23

ROJO, C. \& M. ÁlVAREZ-COBELAS. 2001. Phytoplankton structure and dynamics at a daily temporal scale: response to the thermal overturn. Arch. Hydrobiol., 151: 549-569.

ROJO C., E. ORTEGA-MAYAGOITIA, M. A. RODRIGO \& M. ALVAREZ-COBELAS. 2000a. Phytoplancton structure and dynamics in a semiarid wetland, the National Park "Las Tablas de Daimiel" (Spain). Arch. Hydrobiol., 148:397-419

ROJO C., E. ORTEGA-MAYAGOITIA. \& M. ALVAREZ-COBELAS. 2000b. Lack of pattern among phytoplankton assemblage. Or, what does the exception to the rule mean? Hydrobiologia, 424:133-139

ROUND, F. E., R. M. GRAWFORD \& D. G. MANN. 1990. The diatoms: Biology and Morphology of the genera. Cambridge University Press, Cambridge.

ROTT, E. 1981. Some results from phytoplankton counting intercalibrations. Schweizerische Zeitschrift für Hydrobiologie, 43: 34-62

RUBIO A. \& M. ALVAREZ-COBELAS. 1996. Datos físicos-químicos. En Las Tablas de Daimiel. Ecología acuática y sociedad. Alvarez-Cobelas M \& S Cirujano (eds.): 305-326. Madrid.

SÁNCHEZ-CARRILLO, S., M. ÁLVAREZ-COBELAS, S. CIRUJANO, P. RIOLOBOS, M. MORENO-PÉREZ \& C. ROJO. 2000. Rainfall-driven 
changes in the biovolume of a semi-arid wetland. Verh. Internat. Verein. Limnol., 27: 1690-1694.

SÁNCHEZ-CARRILLO, S. \& M. ÁLVAREZ-COBELAS. 2001. Nutrient dynamics and eutrophication patterns in a semi-arid wetland: the effects of fluctuating hydrology. Water Air Soil Pollution, 127: 12-27.

SCHEFFER, M. 1991. Should we expect strange attractors behind plankton dynamics and if so, should we bother? Journal of Plankton Research, 13: 1291-1305.
SHIEL, R. J., J. D. GREEN \& D. L. NIELSEN. 1998. Floodplain biodiversity: Why are there so many species? Hydrobiologia, 387/388: 39-46.

TELL G. \& V. GARCÍA. 1986. Euglenofita pigmentadas de la Argentina. J. Cramer. Buenos Aires, Argentina.

TROYA A. \& M. BERNUES. 1990. Humedales españoles en la lista del Convenio de Ramsar. ICONA, Ministerio de Agricultura, Alimentación, y Pesca, Madrid. 